Research Paper

\title{
Changes in Left Ventricular Filling in Patients with Persistent Atrial Fibrillation
}

\author{
Franjo Naji, Mihael Pagliaruzzi, Meta Penko, Vojko Kanic, Damijan Vokac ${ }^{\bowtie}$ \\ Department of Cardiology and Angiology, University Clinical Centre, Maribor, Slovenia. \\ $\triangle$ Corresponding author: Franjo Naji, MD, PhD. Department of Cardiology and Angiology, University Clinical Center Maribor, Ljubljanska \\ 5, SI-2000 Maribor, Slovenia. Tel: 0038623212341 E-mail: franjo.naji@yahoo.com. \\ (C) Ivyspring International Publisher. This is an open-access article distributed under the terms of the Creative Commons License (http://creativecommons.org/ \\ licenses/by-nc-nd/3.0/). Reproduction is permitted for personal, noncommercial use, provided that the article is in whole, unmodified, and properly cited.
}

Received: 2013.05.05; Accepted: 2013.09.04; Published: 2013.II.20

\begin{abstract}
BACKGROUND: Former studies showed possible interrelationship between altered ventricular filling patterns and atrial fibrillation (AF).

HYPOTHESIS: Long term persistent AF has a negative impact on left ventricular filling in patients with preserved ejection fraction of left ventricle.

METHODS: Our study was designed as a prospective case control study. We included 40 patients with persistent $A F$ and preserved ejection fraction after successful electrical cardioversion and 43 control patients. Persistent AF was defined as AF lasting more than 4 weeks. Cardiac ultrasound was performed in all patients 24 hours after the procedure. Appropriate mitral flow and tissue Doppler velocities as well as standard echocardiographic measurements were obtained.

RESULTS: There were no significant differences between both groups' parameters regarding age, sex, commorbidities or drug therapy. Analysis of mitral flow velocities showed significant increase of $E$ value in $A F$ group $(0.96 \pm 0.27$ vs. $0.70 \pm 0.14 ; p=0.00 \mathrm{I})$. Tissue Doppler measurements didn't reveal any differences in early diastolic movement, however there was a statistically significant difference in $\mathrm{E} / \mathrm{Em}$ values of both groups, respectively ( $12.0 \pm 4.0$ vs. $9.0 \pm 2.1 ; p=0.00 \mathrm{I})$.

CONCLUSION: Our study shows that in patients with preserved systolic function and persistent AF shortly after cardioversion diastolic ventricular filling patterns are altered mainly due to increased left atrial pressure and not due to impaired diastolic relaxation of left ventricle. Further studies are needed in order to define the interplay between diminished atrial function and impaired ventricular filling.
\end{abstract}

Key words: atrial fibrillation, ventricular filling, diastolic relaxation, echocardiography, electrical cardioversion.

\section{Introduction}

Atrial fibrillation (AF) is the most common rhythm disorder $(1,2)$. It is a risk factor for increased morbidity and mortality, however data showing interrelationship between $\mathrm{AF}$ and heart failure are still conflicting (3-6). Changes in left ventricular filling pattern are connected to left atrial remodeling, which plays an important role in development as well as in perpetuation of $\mathrm{AF}$ (7). It was proven in previous studies, that presence of impaired left ventricular filling increases the risk of recurrent AF after successful electrical cardioversion (EC) as well as after successful ablation of AF $(8,9)$. On the other hand it was recently shown that rapid atrial rhythm induces changes in endothelial parameters which could to some extent impair ventricular contractility and relaxation (10). However, data showing the possible adittional role of diminished atrial function on ventricular diastolic function are still scarce. In this study we tried to evaluate left ventricular filling patterns shortly after cardioversion in patients with persistent $\mathrm{AF}$ and preserved systolic function and establish possible connection between AF and impaired diastolic 
filling.

\section{Methods}

Our study was designed as a prospective case control study. We included 40 patients with persistent AF after successful EC and 43 control patients with long lasting sinus rhythm. We decided to include patients after successful EC primarily to avoid difficulties and possible bias that occurs when trying to measure echocardiographic parameters in presence of arrhythmia. Persistent AF was defined as AF lasting more than 4 weeks. Exclusion criteria were significant valvular disease, known symptoms of heart failure, implanted valve or device, diagnosed cardiomyopathy, ejection fraction $<50 \%$, uncontrolled arterial hypertension (systolic $>180 \mathrm{mmHg}$ and diastolic $>100$ $\mathrm{mmHg}$ ), left bundle brunch block and age $>80$ years. For patients in control group additional exclusion criteria was positive history of AF. All patients scheduled for EC were treated with oral anticoagulant therapy with international normalized ratio between 2 and 3 for at least 4 weeks. Informed consent was obtained in all patients. Study was conducted according to declaration of Helsinki.

EC was performed under sedation with propofol or lorazepam, respectively. Electrodes were placed in standard right parasternal and apical positions or left parasternal and left paravertebral position when needed. Energies up to $360 \mathrm{~J}$ were used in order to perform successful cardioversion. After procedure ECG was recorded. Patients were afterwards monitored for 24 hours.

Before discharge and at least 24 hours after EC transthoracic echocardiography was performed in all patients. Examination was performed with Philips IE 33. Dedicated cardiac probe was positioned in standard parasternal and apical position and appropriate long and short axis parasternal as well as 4 and 2 chamber apical views were obtained. Mitral flow velocity was measured with a pulse wave doppler and sample volume positioned at the tip of mitral leaflets. Peak early (E) transmitral flow velocities were then obtained.

Tissue doppler measurements were recorded from apical 4 chamber view. Pulsed wave Doppler was measured with $5 \mathrm{~mm}$ sample volume positioned at junction of mitral annulus and adjacent septal myocardium. Myocardial early (Em) diastolic velocities were measured and appropriate ratios (E/Em) were immediately calculated. Due to absent or only moderate atrial contractile function late myocardial tissue velocities (Am) were not measured. Median of 5 consecutive complexes was calculated for all obtained measurements.

Patients were divided in AF group and control group. Continuous data are presented as mean $\pm S D$ and categorical as percentages. Differences between both groups' parameters were calculated using Student's t-test or chi-square test. One way ANOVA test was also used to asses differences between continuous parameters, where appropriate. A $p$ value $<0.05$ was considered as statistically significant. Statistical analysis was performed using the IBM SPSS statistical package.

\section{Results}

After inclusion criteria were met, 40 patients with persistent AF after successful EC and 43 controls were included. Data regarding both groups are depicted in Table 1. There were no major differences between both groups in terms of sex, age, or drug therapy. AF patients have got slightly more diabetes, however there was no difference regarding the presence of arterial hypertension in both groups. When comparing echocardiographic measurements between both groups we found statistically significant enlargement of left atrium (LA) in AF group (Figure 1). Analysis of mitral flow velocities showed significant increase of $E$ value as well as decrease of $A$ value in AF group. Tissue Doppler measurements didn't reveal any significant differences, however there was a statistically highly significant difference between $\mathrm{E} / \mathrm{Em}$ values of both groups. Results are presented in Table 2.

Table I. Main patients' characteristics.

\begin{tabular}{llll}
\hline & $\begin{array}{l}\text { Patients with } \\
\mathrm{AF}(\mathrm{n}=40)\end{array}$ & $\begin{array}{l}\text { Control group } \\
(\mathrm{n}=43)\end{array}$ & $\begin{array}{l}\mathrm{P} \\
\text { value }\end{array}$ \\
\hline Sex (male) & $27(67.5 \%)$ & $20(46.5 \%)$ & 0.08 \\
Age (years) & $64.6 \pm 8.8$ & $63.6 \pm 12.9$ & 0.60 \\
Arterial hypertension & $25(62.5 \%)$ & $32(74.4 \%)$ & 0.34 \\
$\begin{array}{l}\text { Diabetes Mellitus } \\
\text { Medications }\end{array}$ & $6(15.0 \%)$ & $1(2.3 \%)$ & 0.05 \\
$\begin{array}{l}\text { ACE inhibitors or ARBs } \\
\text { Beta blockers }\end{array}$ & $27(67.5 \%)$ & $25(58.1 \%)$ & 0.5 \\
\hline
\end{tabular}

Table 2. Echocardiographic measurements of both patients' groups.

\begin{tabular}{llll}
\hline & $\begin{array}{l}\text { Patients with } \\
\mathrm{AF}(\mathrm{n}=40)\end{array}$ & $\begin{array}{l}\text { Control group } \\
(\mathrm{n}=43)\end{array}$ & $\begin{array}{l}\mathrm{P} \\
\text { value }\end{array}$ \\
\hline $\begin{array}{l}\text { Left atrium }(\mathrm{mm}) \\
\begin{array}{l}\text { Intraventricular septum } \\
(\mathrm{mm})\end{array}\end{array}$ & $44.3 \pm 6.5$ & $37.6 \pm 6.3$ & $<0.001$ \\
$\begin{array}{l}\text { Left ventricular diastolic } \\
\text { diameter }(\mathrm{mm})\end{array}$ & $48.5 \pm 1.1$ & $10.7 \pm 1.0$ & 0.30 \\
E value $(\mathrm{m} / \mathrm{sec})$ & $0.96 \pm 0.27$ & $0.70 \pm 0.14$ & $<0.001$ \\
A value $(\mathrm{m} / \mathrm{sec})$ & $0.43 \pm 0.23$ & $0.81 \pm 0.18$ & $<0.001$ \\
Em $(\mathrm{m} / \mathrm{sec})$ & $0.084 \pm 0.022$ & $0.081 \pm 0.025$ & 0.48 \\
E/Em & $12.0 \pm 4.0$ & $9 \pm 2.1$ & $<0.001$ \\
\hline
\end{tabular}




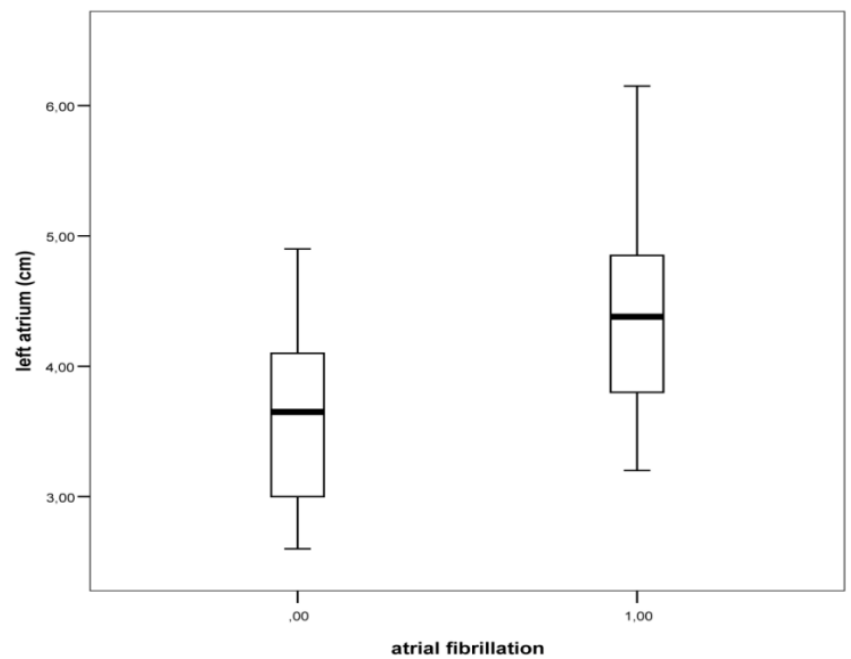

Fig I. The relationship between atrial fibrillation and LA diameter obtained from left parasternal long axis view; box plots represent median levels with $25^{\text {th }}$ and $75^{\text {th }}$ percentiles and whiskers the $5^{\text {th }}$ and $95^{\text {th }}$ percentiles of LA diameters (ANOVA test; $P<0.05$ ) .

\section{Discussion}

Our results show that there is a clear relationship between $\mathrm{AF}$ and changed ventricular filling patterns in patients with recent long-lasting arrhythmia. When compared to control group, it was shown that $\mathrm{E}$ value as well as E/Em ratio were markedly increased in patients with AF. On the other hand, A value was significantly reduced, showing diminished or absent atrial function early after cardioversion. We also found statistically significant enlargement of left atrium (LA) in AF group clearly confirming the presence of long AF duration. Interestingly, major changes occurred in early mitral flow velocity values (E). E value represents velocity of blood stream into the ventricle and is largely dependent upon relaxation of left ventricle as well as atrial pressure. The slower the relaxation of $L V$ or the higher the atrial pressure the higher the peak E velocity (11). Diminished atrial function and consequent elevation of atrial pressure probably altered ventricular filling pattern in our AF patients, however changes in LV relaxation due to tachyarrhythmia could also inflict the E velocity.

The role of Em and E/Em ratio in patients with AF has been validated and described in detail in literature $(12,13)$. Em proves to be excellent marker of lef ventricular relaxation (diastolic function), while $\mathrm{E} / \mathrm{Em}$ ratio reflects left ventricular filling pressure (atrial pressure). We were able to prove that in our patients with AF left atrial pressure is elevated compared to controls; however, we were not able to establish to which extent changes in E/Em ratio were due to diminished ventricular relaxation and to which extent due to loss of atrial contractility. Moreover, we didn't find any changes in mitral annular movement, which would further imply alterations of diastolic relaxation in patients with AF.

Only few previous studies have tried to assess connection between AF and ventricular diastolic filling patterns. Melek et al (14) investigated effects of ECV on conventional Doppler and TDI parameters in patients with persistent AF. Echocardiographic measurements were obtained before, 24 hours and one month after successful ECV. They didn't find any significant changes that would imply improvement of diastolic function parameters after restoration of sinus rhythm except the restoration of atrial contraction; however the observation period was relatively short. On the other hand, in a study conducted by Caputo and colleagues the E/Em ratio was the only significant predictor of recurrent $\mathrm{AF}$ in following year after ECV (9). Ejima et al. tried to evaluate the impact of DD on AF recurrence after successful catheter pulmonary vein isolation (8). They also discovered that impaired diastolic filling was the only independent predictor of AF recurrence after single AF ablation.

All of the above-mentioned studies suggest that patients with more pronounced ventricular filling impairment are in greater risk for AF recurrence. What remains unresolved is whether AF per se can worsen diastolic function of left ventricle. Known patophysiological mechanisms explaining the worsening effect of AF on ventricular function include loss of atrial contractions, irregular ventricular filling, irregular and/or high ventricular rate, however most of these usually take place in acute onset of atrial tachyarrhythmia. On the other hand data showing or explaining possible negative effect of long term persistent $\mathrm{AF}$ on ventricular function is still scarce. Recently Goette et al. showed on mixed human/animal model that persistent $\mathrm{AF}$ as well as rapid atrial pacing downregulate endothelial nitric oxide synthase (eNOS) and elevate levels of inhibitor asymmetric dimethylarginine (ADMA), which are known risk factors for oxidative stress, vascular dysfunction, and congestive heart failure (10). In another study performed by Goette et al. levels of serume aldosterone were measured in patients with chronic persistent AF before and after successful EC. Restoration of sinus rhythm resulted in significant reduction of aldosterone levels, irrespective of other possible confounders (15). Regarding the clinical data only few studies have been conducted for this purpose. Reant et al. measured parameters of systolic and diastolic function in patients after successful ablation in patients with lone AF (16). They proved the presence of impaired diastolic filling in patients with AF compared with healthy controls. Moreover they were also able to confirm the improvement after 12 months since the procedure. They suggested that AF could at least in 
part be the cause rather than consequence of impaired diastolic function. The similar study conducted by the same group showed also the improvement of left ventricular systolic function parameters in patients with paroxysmal AF after successful ablation (17). They demonstrated the existence of early left ventricular abnormalities before the procedure and regression of these abnormalities afterwards. Tops et al. tried to assess the long term effects of catheter ablation for AF on LV strain and strain rate in patients with preserved systolic function. Patients were divided in two groups according to maintenance of sinus rhythm during follow up. They observed significant improvement of LV strain and strain rate in patients with preserved sinus rhythm and deterioration of observed parameters in patients with recurrent $\mathrm{AF}$ (18).

Moreover, in regard to obtained measures in our patients one could speculate that it is AF per se and consequently loss of atrial contractility that leads to elevated left atrial pressure and induces structural changes such as left atrial enlargement and fibrosis. In a recent study performed by Linhart et al. it was shown that left atrial pressure directly measured after transseptal puncture prior to AF ablation correlates with AF recurrence rate in same group of patients (19). Unfortunately we weren't able to perform additional measurements that would give us further insight in arrhythmia induced left atrial tissue derangement.

Our study has several limitations. The studied group was small; however, there was a highly significant statistical difference between both groups regarding E and A value, as well as E/Em ratio. Unfortunately we weren't able to repeat measurements after certain time period, which would give us additional insight into possible connection between $\mathrm{AF}$, cardioversion and diastolic filling patterns. All patients in AF group had recent EC, which could to certain level interfere with relaxation of the ventricle. Nevertheless, all measurements were done at least 24 hours after the EC, consequently reducing the bias of possible temporary cardiac stunning. It was also shown in previous studies that values of diastolic function don't change significantly after EC when compared to values obtained before the procedure (13). Our patients also had concomitant diseases, which could to some extent influence left ventricular relaxation. However, the presence of arterial hypertension and adjoining drug therapy, which were mainly present in both groups, didn't differ significantly in comparative analysis. One could also argue that established atrial function after EC could interfere with obtained measurements. However, since all patients in the AF group had persistent AF, atrial func- tion early after EC (as reflected in A value), was significantly reduced in comparison to control group, therefore minimizing the possible effect on changes in early ventricular diastolic relaxation.

\section{Conclusions}

In conclusion, our study shows that in patients with preserved systolic function presence of persistent AF negatively affects left ventricular filling patterns. Further studies are needed in order to define the interplay between diminished atrial function and impaired ventricular filling.

\section{Competing Interests}

The authors have declared that no competing interest exists.

\section{References}

1. Kannel WB, Wolf PA, Benjamin EJ, Levy D. Prevalence, incidence, prognosis and predisposing conditions for atrial fibrillation: population-based estimates. Am J Cardiol. 1998;82:2N-9N

2. Lloyd-Jones DM, Wang TJ, Leip EP, et al. Lifetime risk for developement of atrial fibrillation: the Framingham Heart Study. Circulation. 2004; 110:1042.

3. Stewart S, Hart CL, Hole DJ, et al. A population-based study of the long -term risks associated with atrial fibrillation: 20 year follow-up of the Renfrew/Paisley study. Am J Med. 2002; 113:359-364.

4. Miyasaka Y, Barnes ME, Gersh BJ, et al. Secular trends in incidence of atrial fibrillation in Olmsted County, Minnesota, 1980 to 2000, and implications on the projections for future prevalence. Circulation. 2006; 114:119-125.

5. Piccini JP, Hammill BG, Sinner MF, et al. Incidence and Prevalence of Atrial Fibrillation and Associated Mortality Among Medicare Beneficiaries, 1993-2007. Circ Cardiovasc Qual Outcomes. 2012; 5:85-93

6. Chamberlain AM, Redfield MM, et al. Atrial fibrillation and mortality in heart failure: a community study. Circ Heart Fail. 2011; 4:740-746.

7. Anne W, Willems R, Holemans P, et al. Self-terminating AF depends on electrical remodeling while persistent AF depends on additional structural changes in a rapid atrially paced sheep model. J Mol Cell Cardiol. 2007; 43:148-58.

8. Ejima K, Shoda M, Arai K, et al. Impact of diastolic dysfunction on the outcome of catheter ablation in patients with atrial fibrillation. Int J Cardiol. 2011; 164:88-93.

9. Caputo M, Urselli R, Capati E, et al. Usefulness of left ventricular diastolic dysfunction assessed by pulsed tissue Doppler imaging as a predictor of atrial fibrillation recurrence after successful electrical cardioversion. Am J Cardiol. 2011; 108:698-704.

10. Goette A, Hammwöhner M, Bukowska A, et al. The impact of rapid atrial pacing on ADMA and endothelial NOS. Int J Cardiol. 2012; 154:141-146.

11. S.F. Nagueh, H.A. Kopelen, M.A. Quinones. Assessment of left ventricular filling pressures by Doppler in the presence of atrial fibrillation. Circulation. 1996; 94:2138-2145.

12. Al-Omari MA, Finstuen J, Appleton CP, Barnes ME, Tsang TS. Echocardiographic assessment of left ventricular diastolic function and filling pressure in atrial fibrillation. Am J Cardiol. 2008; 101:1759-65.

13. Park JH, Marwick TH. Use and Limitations of E/e' to Assess Left Ventricular Filling Pressure by Echocardiography. J Cardiovasc Ultrasound. 2011; 19:169-73

14. Melek M, Birdane A, Goktekin O, et al. The effect of successful electrical cardioversion on left ventricular diastolic function in patients with persistent atrial fibrillation: a tissue Doppler study. Echocardiography. 2007;24:34-39.

15. Goette A, Hoffmanns P, Enayati W, et al. Effect of successful electrical cardioversion on serum aldosterone in patients with persistent atrial fibrillation. Am J Cardiol. 2001; 88:906-909.

16. Reant $\mathrm{P}$, Lafitte $\mathrm{S}$, Jais $\mathrm{P}$, et al. Reverse remodeling of the left cardiac chambers after catheter ablation after 1 year in a series of patients with isolated atrial fibrillation. Circulation. 2005; 112:2896-2903.

17. Reant $\mathrm{P}$, Lafitte $\mathrm{S}$, Bougteb $\mathrm{H}$, et al. Effect of catheter ablation for isolated paroxysmal atrial fibrillation on longitudinal and circumferential left ventricular systolic function. Am J Cardiol. 2009; 103:232-237.

18. Tops LF, Delgado V, Bertini M, et al. Left atrial strain predicts reverse remodeling after catheter ablation for atrial fibrillation. J Am Coll Cardiol. 2011; 57:324-331.

19. Linhart M, Lewalter T, Mittmann-Braun EL, et al. Left atrial pressure as predictor for recurrence of atrial fibrillation after pulmonary vein isolation. J Interv Card Electrophysiol. 2013 Nov;38(2):107-14. 